\title{
Usefulness of the Japan Stroke Scale -Depression Scale- (JSS-D) for the Diagnosis of Post-stroke Depression
}

\author{
Yoshiaki Kaji and Koichi Hirata
}

\begin{abstract}
Background Post-stroke depression (PSD) has a great impact on the quality of life of patients with stroke. The Mini International Neuropsychiatric Interview (MINI) and the Hamilton Depression Scale (HAM- $\mathrm{D}_{17}$ ) are considered the most reliable diagnostic tests for depression. However, both are difficult to conduct in a clinical setting since they require completion of a questionnaire in a limited time period. The Japan Stroke Scale -Depression Scale- (JSS-D) was established by the Japan Stroke Society to evaluate mood disorders following stroke, including PSD. Here, we correlated the results of HAM-D ${ }_{17}$, MINI and JSS-D scores.

Methods We studied 100 stroke patients (mean age: $64.6 \pm 11.6[ \pm \mathrm{SD}]$, range: $32-85$ years) in the subacute phase (2-5 weeks after onset). We determined the correlations between HAM- $\mathrm{D}_{17}$ and JSS-D scores. We used MINI to diagnose PSD, which represented major and minor depression, and compared the results with those of JSS-D.

Results JSS-D scores correlated with those of HAM- $\mathrm{D}_{17}(\mathrm{r}=0.847, \mathrm{p}<0.0001)$. The cutoff value of JSS-D score for PSD was 2.40 . The sensitivity and specificity were 0.950 and 0.988 , respectively.

Conclusion JSS-D is the most valuable diagnostic test for PSD based on its ease of use and reliability for estimating PSD in Japan.
\end{abstract}

Key words: Post-stroke depression, Japan Stroke Scale-Depression Scale, Hamilton Depression Scale, MINI International Neuropsychiatric Interview

(DOI: 10.2169/internalmedicine.47.0512)

\section{Introduction}

Recent studies indicate that cerebrovascular accidents (CVA) are associated with depression in elderly persons, and several investigators have examined poststroke depression (PSD) and vascular depression (1-3). Since it is generally accepted that PSD affects recovery from complications associated with CVA and results in increased mortality (4-6), early detection and appropriate response to PSD are essential (7). However, a simple and reliable method for the diagnosis of PSD is not available at present.

The Mini International Neuropsychiatric Interview (MINI) (8) and the Hamilton Depression Scale $\left(H A M-D_{17}\right)$ (9) are the most reliable tools for the diagnosis of depression. MINI is a structured clinical interview based on the Diagnostic and Statistical Manual of Mental Disorders-Fourth Edition (DSM-IV) (10). On the other hand, HAM-D $\mathrm{D}_{17}$ is used glob- ally as a well-validated tool for assessment of depression. In addition, HAM- $\mathrm{D}_{17}$ is reported to be useful for the diagnosis of PSD (11), however it is difficult to use since it requires completion of a questionnaire in a limited time period.

On the other hand, the Japan Stroke Scale -DepressionScale (JSS-D) (12) was prepared by the Japan Stroke Society as part of a larger system that evaluates stroke patients (13-16), to assess poststroke mood disorders, including PSD (Table). The JSS-D is easier to use clinically compared with other tests for assessment of depression such as HAM-D ${ }_{17}$. In the present study, we evaluated JSS-D for the diagnosis of PSD in Japanese patients.

\section{Materials and Methods}

\section{Subjects}

The study subjects were 100 patients (61 men and 39 
Table 1. The Japan Stroke Scale (Depression Scale) (JSS-D).

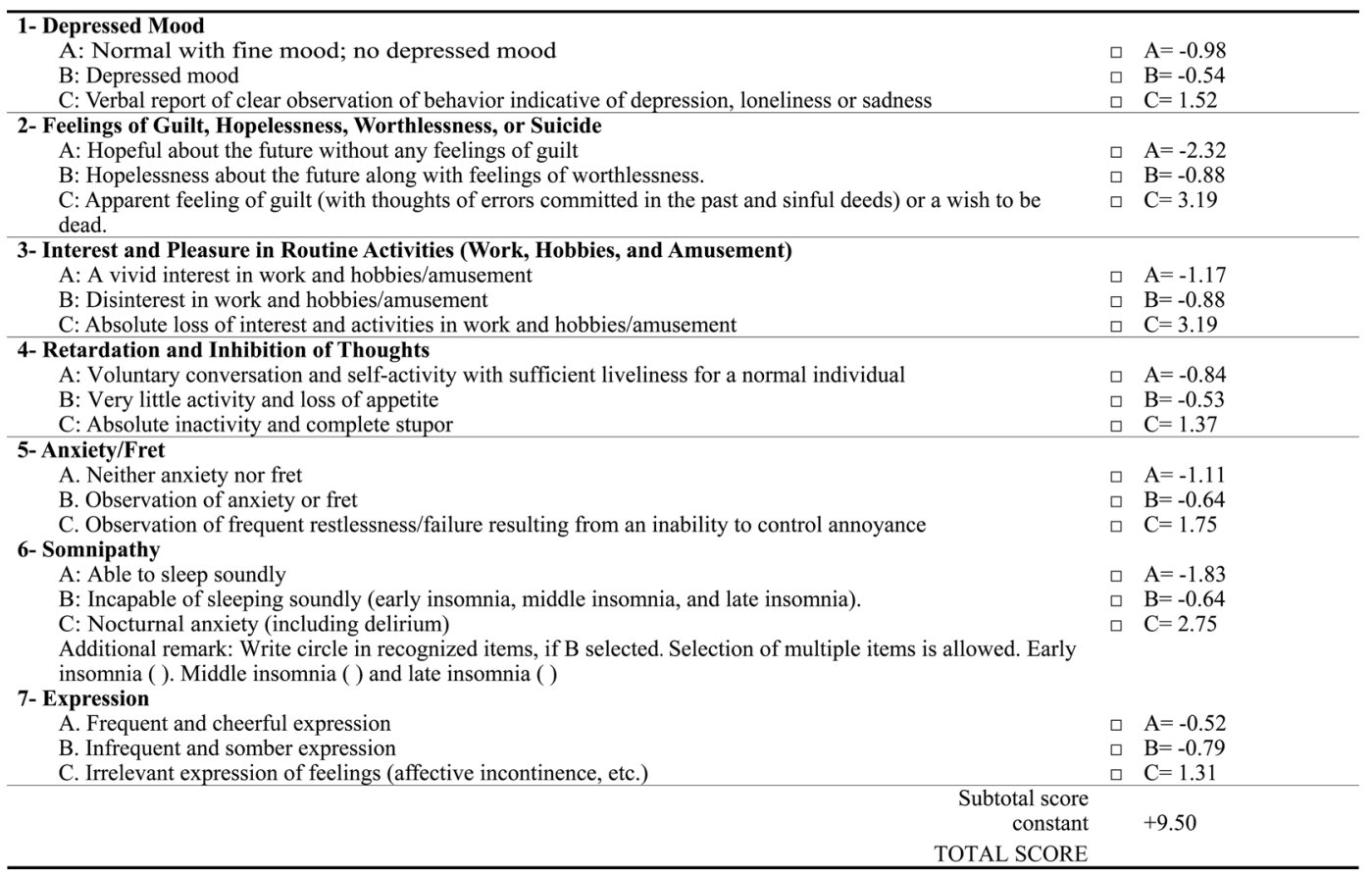

women, mean age: $64.6 \pm 11.6$ years, \pm SD) representing all those admitted consecutively to Dokkyo Medical University Hospital (Department of Neurology) from January 2004 to February 2005 for stroke in the subacute phase (2 to 5 weeks after onset) (Fig. 1).

None of the patients suffered from disturbances of consciousness, and patients with scores less than 21 in the Mini Mental State Examination (MMSE) or with neuropsychological disorders such as aphasia (17) were excluded from this study.

\section{Study protocol}

We used three types of psychological tests for evaluation of PSD, namely, MINI, HAM-D ${ }_{17}$ and JSS-D. The tests were independently conducted in Japanese language by the two authors. We analyzed the correlation between HAM-D ${ }_{17}$ and JSS-D. In addition, we defined both major and minor depression in MINI as PSD, and compared the results with those of JSS-D. Since a clear cutoff score for minor depression is not available in MINI, minor depression was considered present when the HAM-D ${ }_{17}$ score was higher than 10 points. It is reported that a few patients with PSD show depressed mood, feeling of guilt and suicide, compared with functional depression (18). Therefore, we sub-analyzed the correlation between HAM-D $\mathrm{D}_{17}$ and JSS-D in patients with PSD ( $n=69$ ) but without depressed mood, feeling of guilt and suicide.

The MINI, HAM-D $D_{17}$ and JSS-D tests were conducted in an isolated quiet room and each test required about $30 \mathrm{~min}-$ utes for completion. The instructions used for the conduct of the tests were described previously $(19,20)$. Y.K. examined HAD17 and K.H. examined MINI and JSS-D in all patients. Only data of patients who completed all three tests were

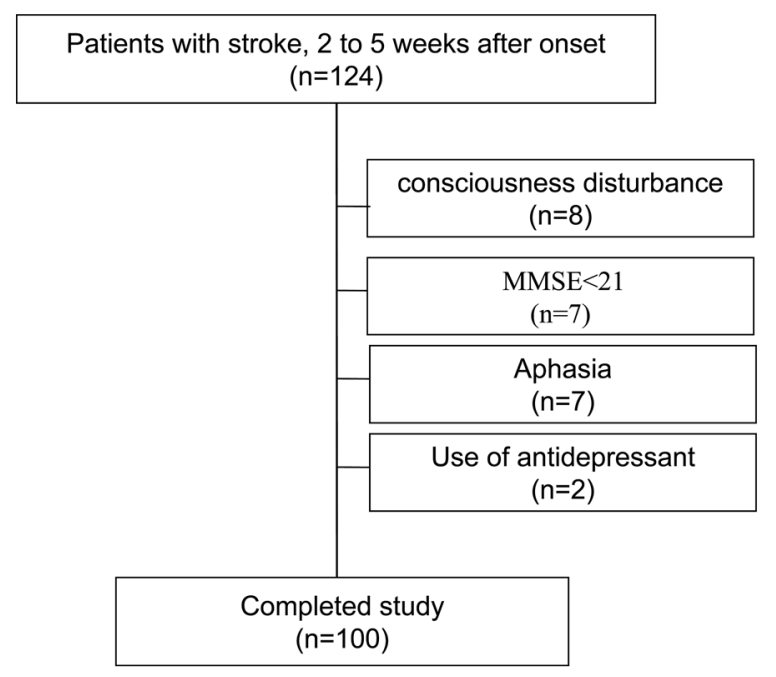

Figure 1. Profile and flow diagram for selection of subjects in the present study.

analyzed in this study. All procedures described in this study were approved by the Ethics Review Committee of Dokkyo Medical University.

\section{Statistical analysis}

Descriptive and analytical statistics were computed using Statcel 2a software (Saitama, Japan). To analyze the correlation between HAM-D $D_{17}$ and JSS-D, we used Spearman's correlation coefficient. A $p$ value $<0.05$ was considered significant. We determined also the cutoff value of JSS-D score for the diagnosis of depression using receiver operating characteristic (ROC) curve analysis, and considered this value as optimal for the diagnosis of PSD under MINI. 


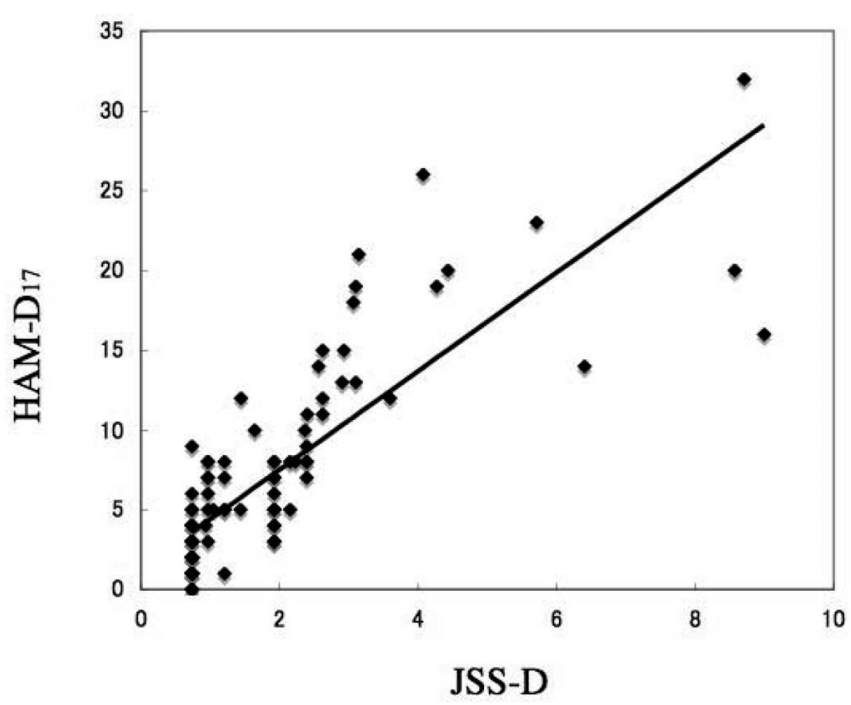

Figure 2. Correlation between HAM-D 17 and JSS-D scores.

\section{Results}

The results of HAM-D $\mathrm{D}_{17}$ correlated significantly with those of JSS-D $(r=0.847, \mathrm{p}<0.0001$, Spearman's correlation coefficient) (Fig. 2). The HAM-D ${ }_{17}$ score was $>11$ points in all patients diagnosed with major and minor depression. Based on our diagnostic criteria for PSD by MINI and HAM-D ${ }_{17}$, the prevalence of PSD was $20.0 \%$ in patients with subacute stroke. In addition, the results of HAM-D $D_{17}$ correlated significantly with those of JSS-D in cases of PSD without depressed mood, feeling of guilt and suicide ( $r=$ $0.724, \mathrm{p}<0.001$, Spearman's correlation coefficient by rank test) (Fig. 3). Furthermore, based on ROC curve analysis of JSS-D, the estimated cutoff score for PSD by JSS-D was 2.40 points. The sensitivity based on this cutoff value was 0.950 and specificity was 0.988 (Fig. 4).

\section{Discussion}

This study was conducted mainly under DSM-IV (MINI). DSM-IV provides the diagnosis of depression while HAMD allows assessment of severity of symptoms.

Several recent studies have investigated $\operatorname{PSD}(2,3,7)$, however the pathophysiological mechanism and prevalence of PSD remain unclear. In previous studies, various psychological tests, such as HAM-D (11), Beck Depression Inventory $(\mathrm{BDI})(21,22)$ and Zung Self-Rating Depression Scale (SDS) (4) were used for the assessment of PSD. However, these tests are used to evaluate depressive state only. On the other hand, JSS-D was established from various useful scales, mainly by eliminating seven items from HAM-D $D_{17}$. JSS-D was established to distinguish PSD from complications related to stroke itself; in fact the seven deleted items are related to physical complaints such as general somatic symptoms. Finally, the items in JSS-D were statistically weighted (12) for stroke patients with neurological symptoms.

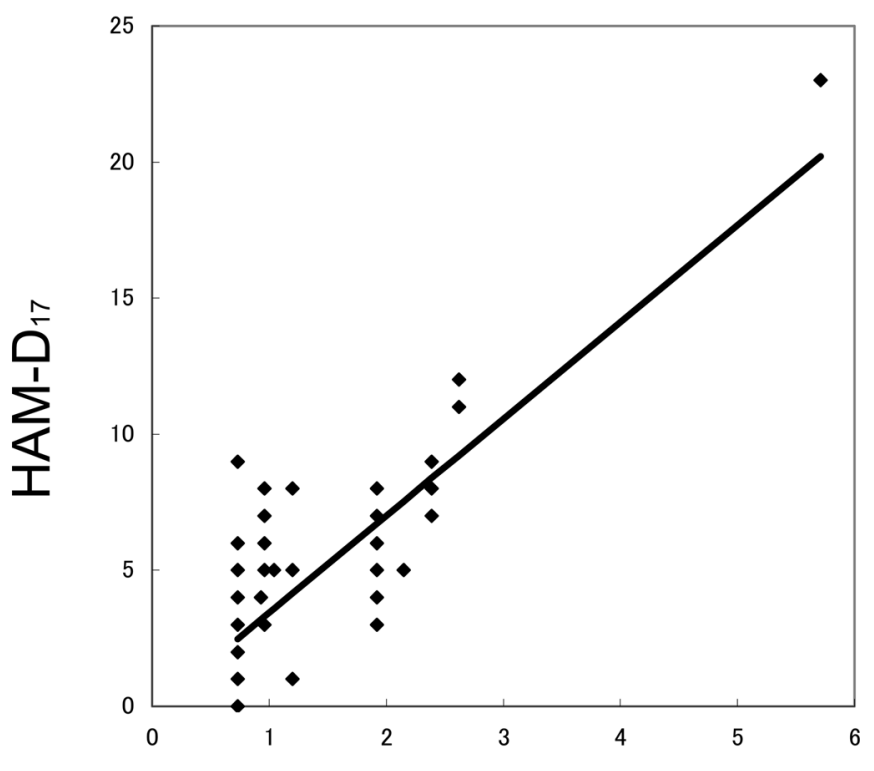

JSS-D

Figure 3. Correlation between HAM-D ${ }_{17}$ and JSS-D for patients without depressed mood, feeling of guilt or suicide.

We evaluated and compared the results of MINI, HAM$\mathrm{D}_{17}$ and JSS-D in this study. Our results showed a significant correlation between HAM-D ${ }_{17}$ and JSS-D scores in Japanese patients with stroke. Although calculation of the results of JSS-D seems difficult, the results can be computed by using a commercially available spreadsheet software, such as Excel. On the other hand, HAM- $D_{17}$ is difficult to use by clinicians since it requires completion of a questionnaire by patients in the outpatient department within a limited time period (20 minutes for the HAM- $D_{17}$, versus 3 minutes only for the JSS-D). In addition to the appropriateness of JSS-D for the diagnosis of PSD, our finding of the significant correlation between JSS-D scores with those of HAM-D $D_{17}$ is important clinically because $H A M-D_{17}$ reflects symptom severity and JSS-D is easier to use than HAM-D ${ }_{17}$.

It is reported that a few patients with PSD show depressed mood and feeling of guilt and are suicidal compared to patients with functional depression (18). The results of HAM-D $D_{17}$ correlated significantly with those of JSS-D in cases of PSD without depressed mood, feeling of guilt and suicide in this study. These data suggest the appropriateness of JSS-D for the diagnosis of PSD rather than functional depression.

With regard to the cutoff level for the diagnosis of minor depression, we usually employ a score of $>10$ in the HAM$D_{17}$ test. We selected this level based on the results of a previous study (23) in which a score $<10$ was considered remission of depression. In fact, in the present study, the HAM-D ${ }_{17}$ score in all patients diagnosed with major and minor depression was $>11$.

The wide use of JSS-D, including the ease of testing, should be promoted. In this pilot study, we found that a score of 2.40 was appropriate for the diagnosis of PSD 


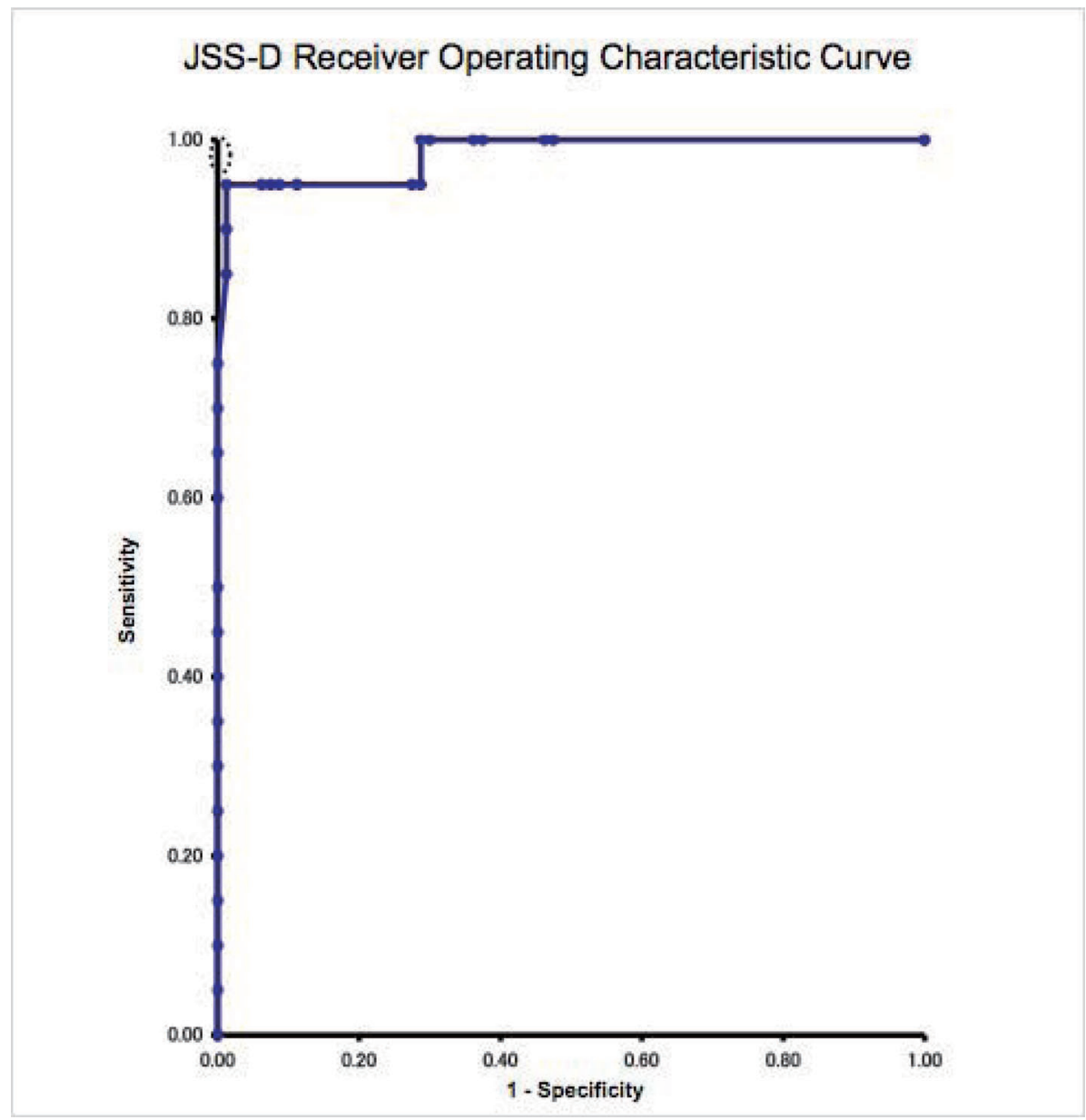

Figure 4. Receiver operating characteristic curve analysis.

based on ROC curve analysis of JSS-D. The limitations of this study are that the number of patients is small, it is a one-off study, and JSS-D has not been validated outside Japan.

\section{Acknowledgement}

We thank Prof. Motoichiro Kato, Department of Neuropsychiatry, Keio University School of Medicine, for the valuable comments and the design of the original version of JSS-D.

\section{References}

1. Fujikawa T, Yamawaki S, Touhouda Y. Incidence of silent cerebral infarction in patients with major depression. Stroke 24: 16311634, 1993.

2. Carson AJ, MacHale S, Allen K, et al. Depression after stroke and lesion location: a systematic review. Lancet 356: 122-126, 2000.

3. Alexopoulus GS, Meyers BS, Young RC, Kakuma T, Silbersweig D, Charlson M. Clinically defined vascular depression. Am J Psychiat 54: 562-565, 1997.

4. Kim JS, Choi-Kwon S. Poststroke depression and emotional incontinence: correlation with lesion location. Neurology 54: 18051810, 2000.

5. Sinyor D, Amato P, Kaloupek DG. Post-stroke depression: relationships to functional impairment, coping strategies, and rehabilitation outcome. Stroke 17: 1102-1107, 1986.

6. Kotila M, Numminen H, Waltimo O, Kaste M. Depression after stroke. Results of The FINNSTROKE Study. Stroke 29: 368-372, 1998.

7. Narushima K, Robinson RG. Stroke-related depression. Curr Atheroscler Rep 4: 296-303, 2002.

8. Sheehan DV, Lecrubier Y, Sheehan KH, et al. The Mini-International Neuropsychiatric Interview (MINI): the development and validation of a structured diagnostic psychiatric interview for DSM-IV and ICD-10. J Clin Psychiatry 59: 22-23, 1998.

9. Hamilton M. A rating scale for depression. J Neurol Neurosurg Psychiatry 56-62, 1960.

10. American Psychiatric Association. Diagnostic and Statistical Manual of Mental Disorders, 4th ed. American Psychiatric Association, Washington DC, 1994.

11. Andersen G, Vestergaard K, Riis J, Lauritzen L. Incidence of poststroke depression during the first year in a large unselected stroke population determined using a valid standardized rating scale. Acta Psychiatr Scand 90: 190-195, 1994.

12. Stroke Scale Committee of The Japan Stroke Society (Preparatory Committee for Emotional Disturbance Scale). Japan Stroke Scale (Emotional Disturbance Scale) <JSS-D/JSS-E > Jpn J Stroke 25: 206-214, 2003 (in Japanese, abstract in English).

13. Gotoh F, Terayama Y, Amano T. Development of a novel, weighted, quantifiable stroke scale. Stroke 32: 1800-1807, 2001.

14. Stroke Scale Committee of The Japan Stroke Society (Preparatory Committee for Emotional Disturbance Scale). Japan Stroke Scale (Motor Function) <JSS-M>. Jpn J Stroke 21: 353-356, 1999 (in Japanese, abstract in English). 
15. Kato M. Development of two weighted scales for depressive symptoms and emotional disorders. International Workshop on Stroke Scales (endorsed by International Stroke Society), Tokyo, Japan, 2000 (Abstract).

16. Stroke Scale Committee of The Japan Stroke Society (Preparatory Committee for Emotional Disturbance Scale). Japan Stroke Scale (Higher Cortical Function) <JSS-H>. Jpn J Stroke 23: 284-291, 2001 (in Japanese, abstract in English).

17. Hasegawa T, Kishi H, Shigeno $K$, Tanemura J, Kusunoki $T$. Three-dimensional structure in language test of aphasia. Folia Phoniatrica 37: 246-258, 1985 (in Japanese, abstract in English).

18. Lipsey JR, Spencer WC, Rabins PV, Robinson RG. Phenomenological comparison of poststroke depression and functional depression. Am J Psychiat 143: 527-529, 1986.

19. Sheehan DV, Lecrubier Y. Mini International Neuropsychiatric Interview, Japanese version 5.00. Seiwa Press, Tokyo, 2003: 7-8 (in
Japanese).

20. Williams J. Structured Interview Guide for the Hamilton Depression Rating Scale, SIGH-D, Japanese version. Seiwa press, Tokyo, 2004: 1-32 (in Japanese).

21. Paolucci S, Gandolfo C, Provinciali L, Torta R, Sommacal S, Toso V; DESTRO Study Group. Quantification of the risk of post stroke depression: the Italian multicenter observational study DESTRO. Acta Psychiatr Scand 112: 272-278, 2005.

22. Sinyor D, Amato P, Kaloupek DG. Post-stroke depression. Relationships to functional impairment, coping strategies, and rehabilitation outcome. Stroke 17: 1102-1107, 1986.

23. Roose SP, Sackeim HA, Krishnan KR, et al. Old-Old Depression Study Group. Antidepressant pharmacotherapy in the treatment of depression in the very old: A randomized, placebo-controlled trial. Am J Psychiatry 161: 2050-2059, 2004.

(C) 2008 The Japanese Society of Internal Medicine http://www.naika.or.jp/imindex.html 